\title{
Development of Optimization Software to Solve Practical Packing and Cutting Problems
}

\author{
Vladislav A. Chekanin*, Alexander V. Chekanin \\ Moscow State University of Technology «STANKIN» \\ Moscow, Russian Federation \\ vladchekanin@rambler.ru*, avchekanin@rambler.ru \\ *Corresponding author
}

\begin{abstract}
The paper describes a developed optimization software intended to solve various practical problems of recourse allocation. In a basis of the software lays a designed class library for solving different recourse allocation problems including packing and cutting problems. Due to belonging of all packing problems to the class of NP-completed problems they cannot be solved in polynomial time depending on their size. To solve such problems are usually used metaheuristic algorithms of multicriteria optimization. The designed class library allows to solve all packing and problems using a variety of metaheuristic algorithms which makes the developed software universal with respect to types of solved problems and algorithms used.
\end{abstract}

Keywords-optimzation software; class library; metaheuristc algorithms; evolutionary algorithms; packing problem

\section{INTRODUCTION}

The packing and cutting problems are ones of the actual classical optimization problems. They combine a large set of various practical problems related with finding of the most optimal allocation of resources of one type (named by objects) into resources of another type (named by containers). In general case in most of packing problems it is necessary to pack all given objects into minimal number of containers or it is necessary to find a maximal number of objects which can be placed into a given set of free containers. The first case is known as input minimization problem and the second - as output maximization problem [1].

The first typology of cutting and packing problems was offered by H. Dyckhoff in 1990 [2]. According to this typology to a class of cutting and packing problems are related such problems as cutting stock problem, trim loss problem, nesting problem, knapsack problem, bin packing problem, pallet and vehicle loading, partitioning problem, memory allocation problem and other.

The full survey of packing problems was prepared by G. Wascher, H. Haubner and H. Schumann in 2007 [1]. Among the most popular types of packing problems in industry and manufacturing can be distinguished orthogonal bin packing problems, cutting stock problems and knapsack problems. The most frequently the packing problems are occurred in solution of such practical problems as waste minimization in cutting, pallet building in logistics, filling up containers (including truck, ship and air cargo loading), traffic and calendar planning and many others [3-6]. Optimization of the packing problems leads to more efficient usage of free storage spaces in practice $[1,7]$.
All optimization packing and cutting problems are belonged to NP-completed problems and they cannot be solved in a time that polynomial depends on the dimension of the problem [8]. The effective approach to solve NP-completed problems is application of various metaheuristic optimization methods of artificial intelligence to them. The most effective methods that used for solving all the packing and cutting problems are evolutionary algorithms among which are the genetic algorithm, the annealing algorithm, the ant colony algorithm, as well as their modified and combined variants [6, 9-11].

\section{Class LibRaRy FOR THE PACKING PROBLEMS}

To solve of various packing and cutting problems via a variety of heuristic and evolutionary algorithms of discrete optimization has been developed an unified class library that is characterized by the following features:

- $\quad$ the possibility of solving different types of cutting and packing problems;

- the ability of solving the packing problems of various dimension;

- the possibility of expanding the class library by appending into it new types of objects to be packed;

- the possibility of expanding the class library by appending into it new metaheuristic optimization algorithms for solving the problems.

Unification of the class library allowing to use it to solve a variety of packing problems, is achieved by using of a common scheme for coding solutions as well as by using of universal packing representation model (the node model) for description of resulting placements [12-14]. In this case a position of each placed into a container object is described only with one point that called by node. All solutions are represented in the form of coded strings (named by chromosomes) which contain sequences of objects to be selected for allocation in containers.

The class library for the packing problems was developed using the object-oriented approach, the major advantages of which include encapsulation of methods and data in classes, overloading of methods, modeling of relationships between objects in an environment very close to reality, naturalness and distinctness of the programming process, as well as simplicity of support and further modifying of the program code.

The class library is developed with the high-level objectoriented programming language $\mathrm{C}++$. 
The developed class library for solving the packing and cutting optimization problems is shown as the UML diagram

on Fig. 1.

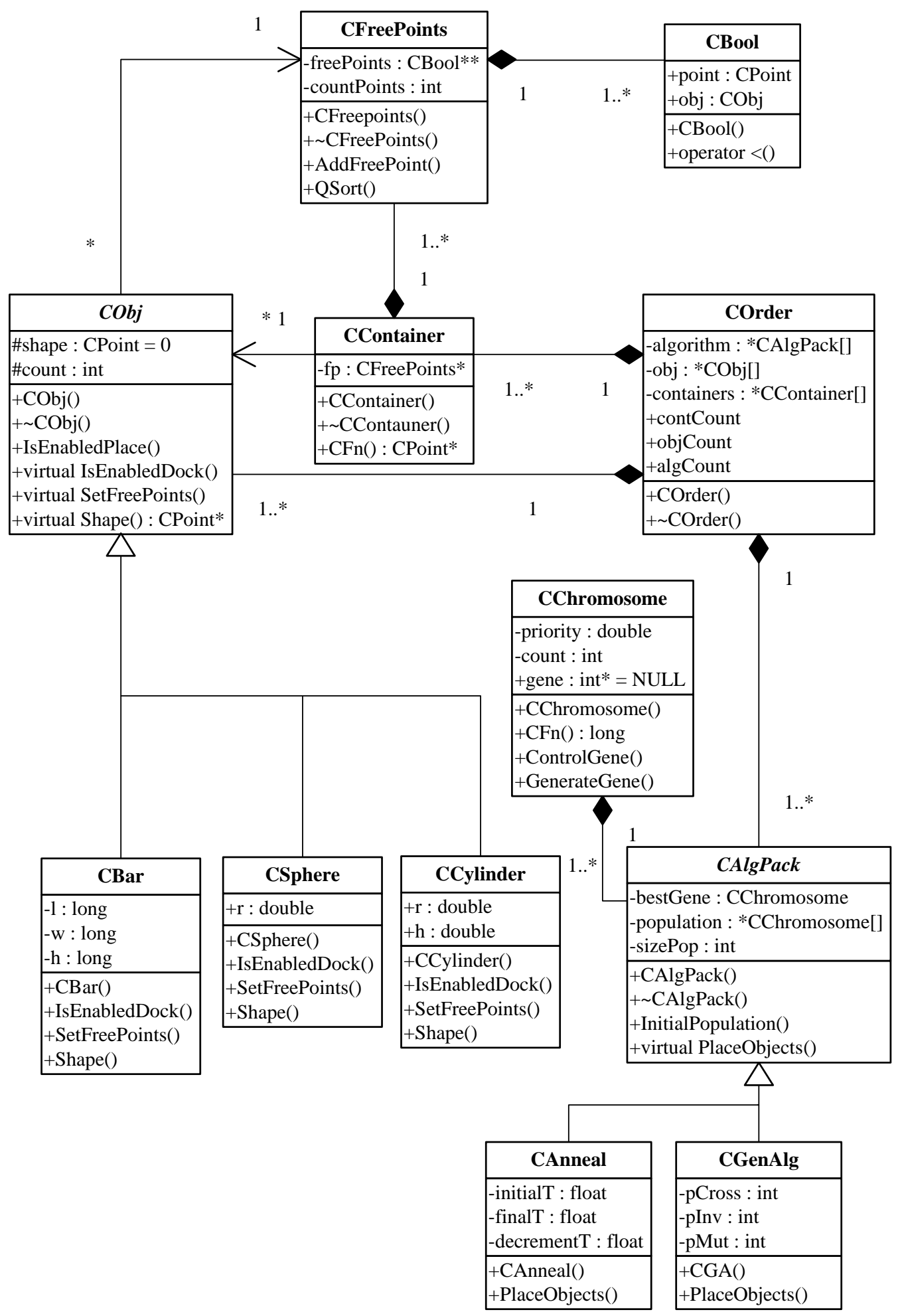

FIGURE I. UML DIAGRAM OF THE DEVELOPED CLASS LIBRARY FOR PACKING AND CUTTING PROBLEMS

In a basis of the class library are class of a problem, abstract class of objects and abstract class of algorithms. The class of a problem COrder is intended for managing all operations related with solving the packing problem. It contains information 
about the type of problem to be solved, its parameters, constraints on objects to be packed and arrays of references to the classes of objects and containers. Additionally, this class contains information about selected optimization algorithms for solving the given problem.

To describe objects is intended an abstract class of objects CObj. From this class are inherited a class of an orthogonal object CBar, a class of a cylinder CCylinder and a class of a sphere CSphere. The class CBar allows describe such objects as line, rectangle and any dimensional parallelepiped including normal three-dimensional parallelepiped and cube.

The abstract class of objects CObj contains the following virtual functions:

- function returning the size of an object Shape();

- function defining possibility of placement of an object into a container IsEnabledPlace();

- function updating the set of nodes in a container SetFreePoints().

The abstract class of objects CObj contains information about the geometrical (e.g. linear dimensions), physical (e.g. weight) and other parameters (for example, cost, fragility, possibility of rotation) of objects. This class allows expanding the class library by including into it new types of objects to be packed during solving an allocation problem.

To describe algorithms for solving the packing and cutting problems is intended an abstract class of algorithms CAlgPack. This class contains two virtual functions:

- function creating an initial population of solutions InitialPopulation();

- function solving a packing problem PlaceObjects().

From this class are inherited a class of a genetic algorithm CGenAlg and a class of an anneal simulated algorithm CAnneal. The class CGenAlg contains the probabilities of implementing of the basic genetic operators (the crossover operator pCross, the mutation operator pMut and the inversion operator pInv). The class of an annealing algorithm CAnneal contains the initial temperature initialT, the final temperature finalT and the degree of cooling decrement $\mathrm{T}$.

The class of algorithms contains a size of a population of solutions sizePop, the best found solution bestGene and the population of solutions in the form of an array of pointers to a class of a solution CChromosome. Usage of the abstract class of algorithms CAlgPack allows to expand the class library by appending into it a set of new modified and combined heuristic and evolutionary algorithms for solving the packing and cutting problem.

The class of a container CContainer contains a method CFn() for estimation of free unfilled spaces of a container as well as a pointer on a class of nodes CFreePoints described a packing.

Using the designed class library was developed an applied software "Packer" intended to solve the cutting and packing optimization problems.

On Fig. 2 and Fig. 3 are shown windows with initial settings of a new packing problem and the corresponding default settings of the classical genetic algorithm used for optimization of the considered packing problem.

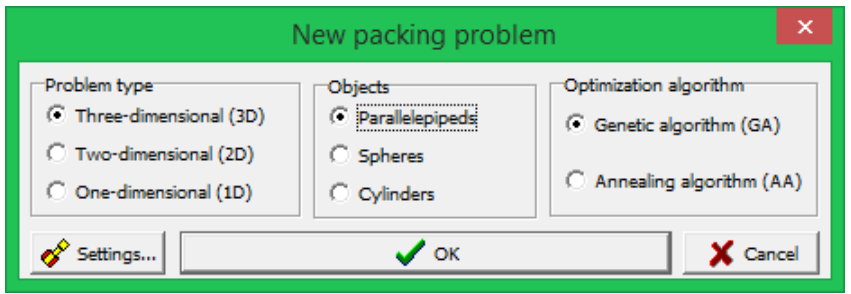

FIGURE II. PARAMETERS OF A NEW PACKING PROBLEM

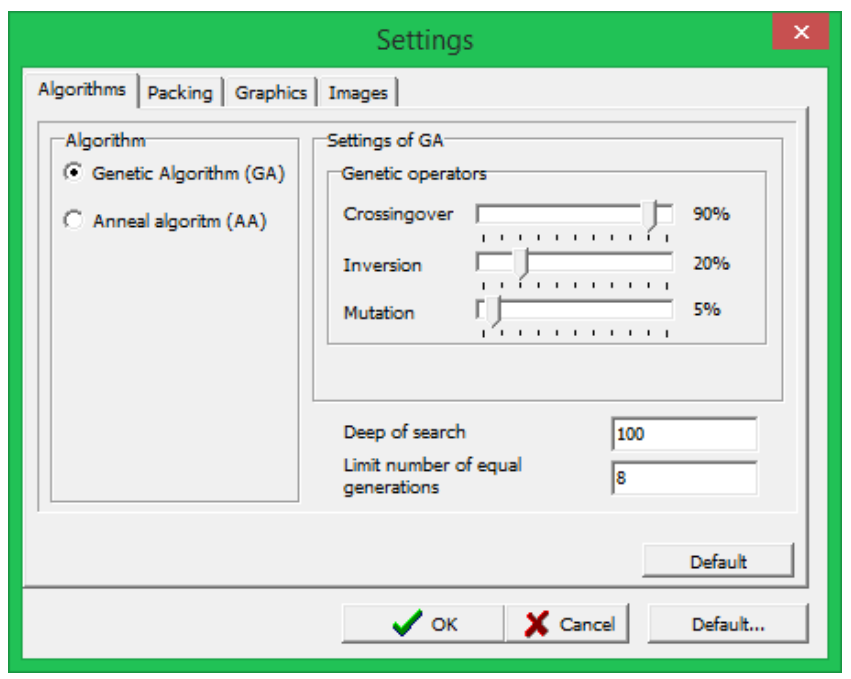

FIGURE III. SETTINGS OF OPTIMIZATION ALGORITHMS

Windows with the parameters of a solved three-dimensional packing problem are shown on Fig. 4. The main window of the software Packer with the result of solving of the considered orthogonal packing problem is shown on Fig. 5.

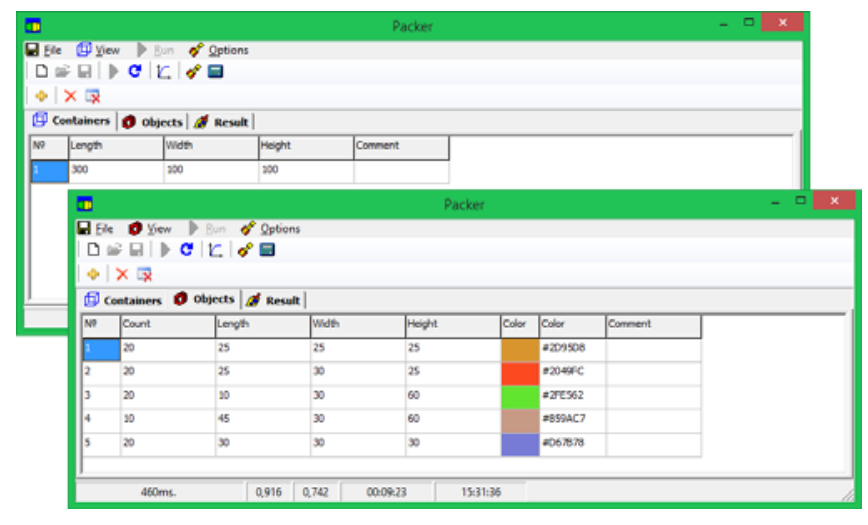

FIGURE IV. PARAMETERS OF A THREE-DIMENSIONAL ORTHOGONAL PACKING PROBLEM 


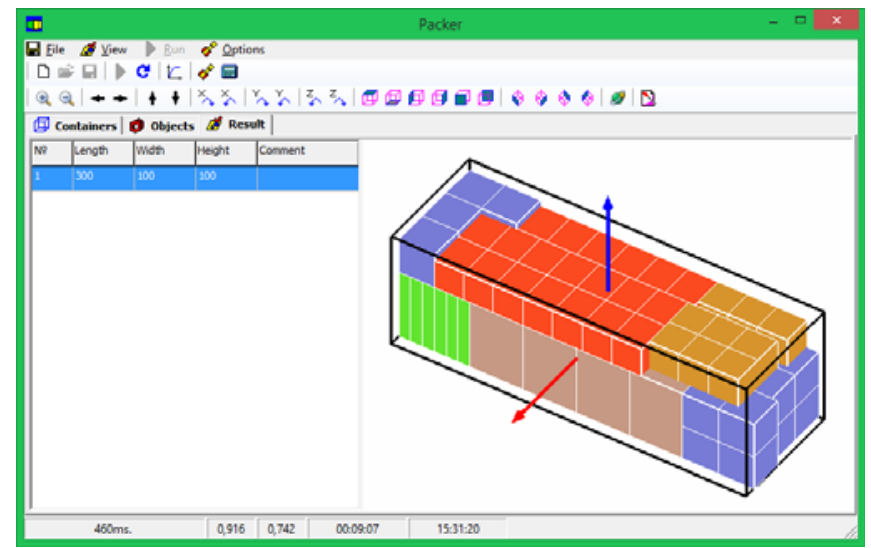

FIGURE V. MAIN WINDOW OF THE SOFTWARE “PACKER” WITH A FOUND SOLUTION

\section{CONCLUSION}

The designed unified class library can be used in solving of a variety of optimization problems of resource allocation including such problems as cutting stock problems, twodimensional and three-dimensional orthogonal bin packing problems, knapsack problems, strip packing problems and many other major in industry and manufacturing problems. The developed applied optimization software based on the class library can be used to solve cutting and packing problems as well as for analysis of effectiveness of various known and newly created metaheuristic algorithms applied for optimization of the considered problem.

\section{ACKNOWLEDGMENTS}

This work was carried out with the financial support of the Ministry of Education and Science of Russian Federation in the framework of the state task in the field of scientific activity of MSTU «STANKIN» (No. 2014/105).

\section{REFERENCES}

[1] G. Wascher, H. Haubner and H. Schumann H, “An improved typology of cutting and packing problems”, European Journal of Operational Research, vol. 183(3), 2007, pp. 1109-1130.

[2] H. Dyckhoff, “A typology of cutting and packing problems”, European Journal of Operational Research, vol. 44, 1990, pp. 145-159.

[3] A. Bortfeldt and G. Wascher, "Constraints in container loading - A state-of-the-art review”, European Journal of Operational Research, vol. 229(1), 2013, pp. 1-20.

[4] A. V. Chekanin and V. A. Chekanin, "Effective data structure for the multidimensional orthogonal bin packing problems", Advanced Materials Research, vol. 962-965, 2014, pp. 2868-2871.

[5] A. Lodi, S. Martello, and M. Monaci M, “Two-dimensional packing problems: A survey”, European Journal of Operational Research, vol. 141(2), 2002, pp. 241-252.

[6] M. C. Riff, X. Bonnaire and B. Neveu, "A revision of recent approaches for two-dimensional strip-packing problems”, Engineering Applications of Artificial Intelligence, vol. 22(4-5), 2009, pp. 823-827.

[7] V. A. Chekanin and A. V. Chekanin, "Improved data structure for the orthogonal packing problem”, Advanced Materials Research, vol. 945949, 2014, pp. 3143-3146.

[8] M. Garey and D. Johnson, Computers Intractability: A Guide to the theory of NP-completeness, San Francisco: W.H.Freeman, 1979.
[9] A. V. Chekanin and V. A. Chekanin, "Efficient algorithms for orthogonal packing problems”, Computational Mathematics and Mathematical Physics, vol. 53(10), 2013, pp. 1457-1465.

[10] K-H. Loh, B. Golden, and E. Wasil, “A weight annealing algorithm for solving two-dimensional bin packing problems", Operations Research/Computer Science Interfaces, vol. 47, 2009, pp. 121-146.

[11] V. A. Chekanin and A. V. Chekanin, "Development of the multimethod genetic algorithm for the strip packing problem”, Applied Mechanics and Materials, vol. 598, 2014, pp. 377-381.

[12] T. G. Crainic, G. Perboli, and R. Tadei, "Extreme point-based heuristics for three-dimensional bin packing”, INFORMS, Journal on Computing, vol. 20(3), 2008, pp. 368-384.

[13] A. V. Chekanin and V. A. Chekanin, "Improved packing representation model for the orthogonal packing problem”, Applied Mechanics and Materials, vol. 390, 2013, pp. 591-595.

[14] V. A. Chekanin and A. V. Chekanin, "Multilevel linked data structure for the multidimensional orthogonal packing problem”, Applied Mechanics and Materials, vol. 598, 2014, pp. 387-391. 